\title{
Condition based maintenance model for a production deteriorating system
}

\author{
Phuc Do Van and Christophe Bérenguer
}

\begin{abstract}
This paper addresses a condition based maintenance model taking into account both maintenance cost and production capacity aspects for a single unit production system whose condition is monitored by inspection relying on an aperiodic inspection schedule. In order to assess the performance of the proposed maintenance policy, maintenance cost per unit of product is introduced and calculated analytically. A numerical example of a multi state production system is finally introduced to illustrate the performance of the proposed policy, as well as the advantages of proposed evaluation approach.
\end{abstract}

\section{INTRODUCTION}

Health monitoring equipments providing information about the system condition have evolved rapidly over the past decade. They are useful tools in maintenance decisionmaking. Based on the monitoring information, different condition-based or predictive maintenance policies which lead to avoid failure occurrence at the lowest cost can be performed, see for example [1], [3], [6]. In the context of deteriorating production system [8], [12], the deterioration causes lower production rates (therefore higher production cost per item) and lower product quality. Preventive maintenance is one of the efficient tools to increase the reliability of the production system. Without an effective maintenance program, the system might be down for significant amounts of time. This means that the effective production rate decreases significantly and the system might not be able to cope with demand.

In many preventive maintenance policies, the decision parameters (preventive threshold, inspection schedule,...) are reached based on only the maintenance cost per unit of time criterion without taking into consideration of the production capacity which may depend on the system condition, e.g. the degradation level [8]. Hence, a lowest maintenance cost rate policy doesn't guaranty an optimal production rate and a higher maintenance cost rate policy may lead to a better production rate. The first objective of this paper is to propose a condition based maintenance model taking into account both maintenance cost rate and production rate. This joint consideration can lead to a better maintenance decision-making. Moreover, in condition based maintenance practice, the monitoring inspections are usually performed at regular intervals. However, it may not be always profitable to systematically inspect the system, especially if the inspection procedure is costly. The present paper proposes to use an

Charles Delaunay Institute - STMR UMR CNRS 6279, Systems Modeling and Dependability Group, Troyes university of technology, 12 rue Marie Curie, BP 2060-10010 Troyes cedex, France van_phuc. do@utt . fr, christophe.berenguerdutt.fr aperiodic inspection policy based on the residual useful life (RUL) of the system.

From a practical point of view, simulation method is often used for the calculation of the maintenance cost rate [7], [10]. This simulation method is usually easy to implement, however it often requires a long simulation time. The second goal of this paper is to develop an analytical approach, based on renewal theory and semi-regenerative technics, proposed recently in [6]. A Gamma stochastic process is used to describe the system deterioration behaviors.

This paper is organized as follows. The first section is devoted to the description of the system characteristics and maintenance model. The aperiodic inspection policy is also considered. The second section focuses on the optimisation of maintenance policies. An analytical calculation of the maintenance cost and production capacity function is established. A simple numerical example is introduced in this section to illustrate the proposed maintenance model. Some numerical results are in addition discussed here. Finally, the last section presents the conclusions drawn from this work.

\section{MODEL DESCRIPTION AND ASSUMPTIONS}

For multi-state production systems such as manufacturing production lines and power generation installations, the performances output of interest is not only the availability but also the production capacity which can settle on different levels (e.g. $100 \%, 90 \%, 80 \%$ of the nominal capacity) depending on the system conditions [4], [8], [9].

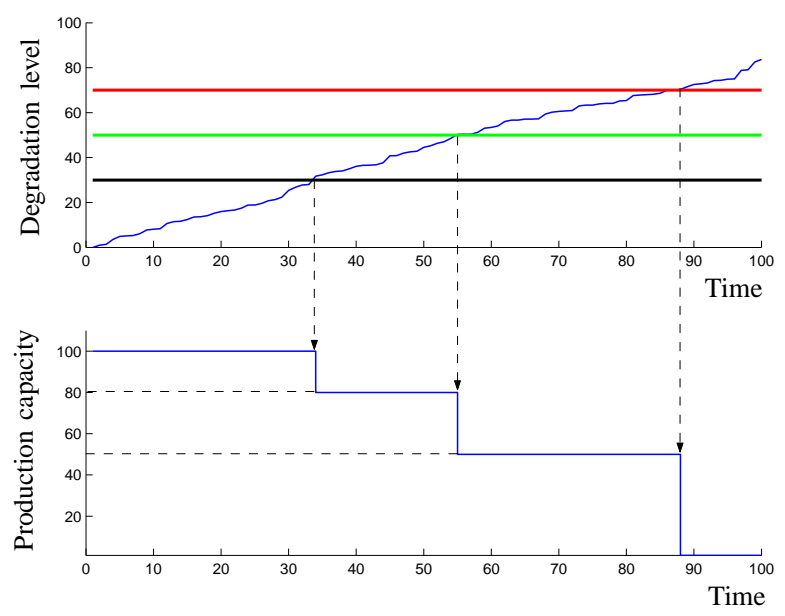

Fig. 1. Illustration of degradation level and corresponding production capacities. 


\section{A. General assumptions}

We consider a single unit system in which its condition at time $t$ can be summarized by a random ageing variable $X_{t}$. In the absence of repair or replacement actions, $X_{t}$ is an increasing process. The ageing variable $X_{t}$ can be e.g. the measure of a physical parameter linked to the resistance of a structure (height of a dike, length of a crack). Moreover, we suppose that the following assumptions are verified.

- The initial state $X_{0}$ is 0

- The system is in failed if the ageing variable is greater than a level $L$. The threshold $L$ can be seen as a deterioration level which must not be exceeded for economical or security reasons.

- In the allowed operation interval (i.e. from 0 to L), $N$ different operation modes are considered. A unique production (or treatment) capacity $S_{i}$ corresponds to each operation mode (or state) $i$ according to the degradation level of the system. More precisely:

- $X_{t}<L_{1}$ : system is in nominal state denoted state 1 in which the system is functioning with nominal production capacity $S_{1}$

- $L_{1} \leq X_{t}<L_{2}$ : system is in operational state (state 2) in which the production capacity is $S_{2}<S_{1}$.

- $\ldots$

- $L_{N-1} \leq X_{t}<L_{N}=L$ : system is still functioning but badly (state $N$ ), production capacity for this case is $S_{N}<S_{N-1}$

The system degradation behavior and corresponding states are illustrated in Fig. 1.

\section{B. Deterioration modelling}

Gamma processes have been widely used to describe the degradation of systems in structural engineering [13]. Herein, the deterioration between two maintenance operations is assumed to evolve like a Gamma stochastic process $\left(\tilde{X}_{t}\right)_{t \geq 0}$, with the following characteristics:

- $\tilde{X}_{0}=0$;

- $\left(\tilde{X}_{t}\right)_{t \geq 0}$ has independent increments;

- for all $0 \leq l<t$, the random increment $\tilde{X}_{t}-\tilde{X}_{l}$ follows a Gamma probability density with shape parameter $\alpha(t-l)$ and scale parameter $\beta$ :

$f_{\beta, \alpha(t-l)}(x)=\frac{1}{\Gamma(\alpha(t-l))} \beta^{\alpha(t-l)} x^{\alpha(t-l)-1} e^{-\beta x} \mathcal{I}_{\{x \geq 0\}}$,

where $\alpha$ is a positive real number.

The mean deterioration speed and its variance are $\alpha \beta$ and $\alpha \beta^{2}$ respectively. Various deterioration behaviors can be modeled by changing the couple of parameters $\alpha, \beta$.

\section{Maintenance model}

A maintenance policy relies on two main decisions: when to take (preventively/correctively) maintenance actions and when to inspect.

In the framework of condition based maintenance strategies, a preventive replacement threshold $M$ is usually considered. According to the degradation level $X_{t}$, the maintenance decision is the following:

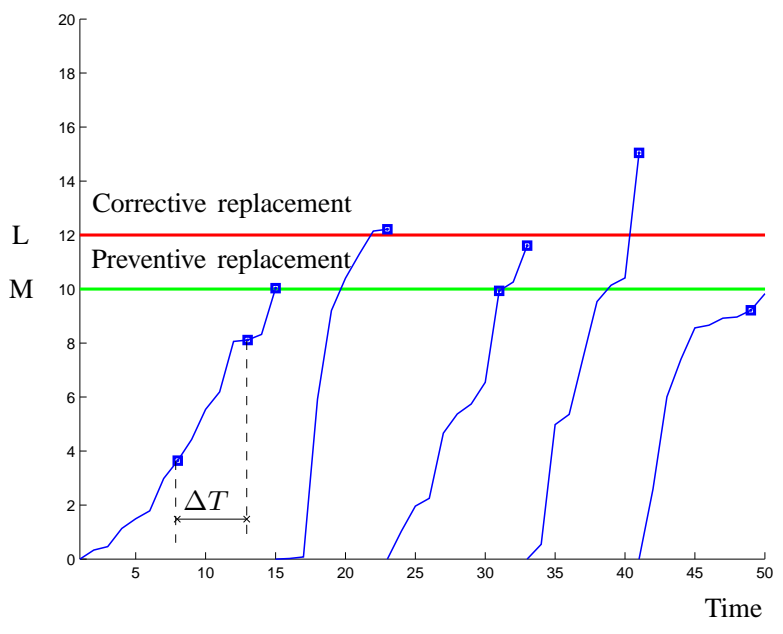

Fig. 2. Illustration of degradation behavior and maintenance policy.

- if $X_{t}<M$ then no maintenance action is performed;

- if $L>X_{t} \geq M$ (system is still functioning), then a preventive maintenance action is performed and a cost $C_{p}$ is incurred ;

- if $X_{t} \geq L$ (system failed), then a corrective maintenance action is performed and a cost $C_{c}\left(C_{p} \leq C_{c}\right)$ is incurred. An additional cost is incurred by the time $d(t)$ elapsed in the failed state at a cost rate $C_{d}$ which may correspond to production lost per unit of time;

For both cases (preventive, corrective maintenance action), the system is considered as good as new (the degradation level after maintenance is assumed to equal 0) The illustration of degradation evolution and maintenance policy is shown in Fig. 2.

The inter-inspection interval length $\Delta T$ (see Fig.2) is recently a popular issue to researchers. This inter-inspection interval length can be fixed regardless of the degradation level, e.g. [11], or aperiodic and deteriorating-dependant via an inspection scheduling linear [6], or non-linear [1] function with respect to the deterioration level. Quantilebased inspection or RUL (Residual Useful Life) based inspection has been recently introduced, see [2], [5], [10], [14]. The latter seems to be very promising especially in the context of condition based maintenance.

Quantile-based inspection policy The main idea is that the next inspection time is chosen such that the probability of the failure of the system before the next inspection remains lower than a limit $Q(0<Q<1)$ to be optimized. If we let $T_{i}$ denote the time at which the system is inspected, the corresponding degradation level of the system is $X_{T_{i}}$, the next inspection time is then determined by:

$$
T_{i+1}=T_{i}+m\left(X_{T_{i}}\right),
$$


with,

$$
\begin{aligned}
& m\left(X_{T_{i}}\right) \\
& =\max \left(1, \sup \left\{\Delta T: P\left(X_{T_{i}+\Delta T} \geq L \mid X_{T_{i}}\right) \leq Q\right\}\right),
\end{aligned}
$$

where:

$$
\begin{aligned}
P\left(X_{T_{i}+\Delta T} \geq L \mid X_{T_{i}}\right) & =P\left(X_{T_{i}+\Delta T}-X_{T_{i}} \geq L-X_{T_{i}}\right) \\
& =\int_{L-X_{T_{i}}}^{\infty} f_{\beta, \alpha\left(t-T_{i}\right)}(x) d x \\
& =1-\int_{0}^{L-X_{T_{i}}} f_{\beta, \alpha\left(t-T_{i}\right)}(x) d x .
\end{aligned}
$$

It is clear that $m\left(X_{T_{i}}\right)$ depend on the degradation level, the failure threshold $L$ and the parameter $Q$. Different forms of this inter-inspection time function $m($.$) for a case study$ are illustrated in Fig. 3, 4 and 5.

Assume finally that the cost for each inspection is $C_{i}$.

\section{OPTIMIZATION OF THE MAINTENANCE POLICIES}

\section{A. Cost based criterion}

The objective cost function to minimize is the long run $s$-expected cost rate $E C_{\infty}$. The cumulative maintenance cost at time $t$ is

$$
C(t)=C_{i} \cdot N_{i}(t)+C_{p} \cdot N_{p}(t)+C_{c} \cdot N_{c}(t)+C_{d} \cdot d(t),
$$

where:

- $N_{i}(t)$ is the number of inspection in $[0, t]$

$$
N_{i}(t)=\sum_{n \subseteq \mathcal{N}} \mathcal{I}_{\left\{t \leq T_{n}\right\}}
$$

- $N_{p}(t)$ is the number of preventive replacement in $[0, t]$

$$
N_{p}(t)=\sum_{n \subseteq \mathcal{N}} \mathcal{I}_{\left\{t \leq T_{n}\right\}} \mathcal{I}_{\left\{M \leq X_{T_{n}^{-}}<L\right\}}
$$

- $N_{c}(t)$ is the number of corrective replacement in $[0, t]$

$$
N_{c}(t)=\sum_{n \subseteq \mathcal{N}} \mathcal{I}_{\left\{t \leq T_{n}\right\}} \mathcal{I}_{\left\{X_{T_{n}^{-}} \geq L\right\}}
$$

- $d(t)$ is the time passed in a failed state in $[0, t]$

$$
d(t)=\int_{0}^{t} \mathcal{I}_{\left\{X_{l} \geq L\right\}} d l .
$$

The long run expected cost rate is:

$$
\begin{aligned}
E C_{\infty} & =\lim _{t \rightarrow \infty}\left[\frac{E[C(t)]}{t}\right] \\
& =C_{i} \lim _{t \rightarrow \infty}\left[\frac{E\left[N_{i}(t)\right]}{t}\right]+C_{p} \lim _{t \rightarrow \infty}\left[\frac{E\left[N_{p}(t)\right]}{t}\right] \\
& +C_{c} \lim _{t \rightarrow \infty}\left[\frac{E\left[N_{c}(t)\right]}{t}\right]+C_{d} \lim _{t \rightarrow \infty}\left[\frac{E[d(t)]}{t}\right] .
\end{aligned}
$$

By using renewal theory, it is shown in [3] and [6] that the limit in (5) is equal to the ratio of the expected cost incurred in a renewal cycle divided by the expected length of a renewal cycle.

$$
E C_{\infty}=\lim _{t \rightarrow \infty}\left[\frac{E[C(t)]}{t}\right]=\frac{E[C(H)]}{E[H]},
$$

where $H$ is the first replacement date.

In maintenance optimisation, this cost rate is usually used. However in the framework of production system, productivity takes an important role and should be taken into account in the optimisation procedure. In the next paragraph, we consider the optimisation problem of multicriteria: maintenance cost and productivity.

\section{B. Cost and productivity based criterion}

The cumulative production capacity at time $t$ is:

$$
\operatorname{Pr}(t)=\sum_{i=1}^{N} D_{i}(t) . S_{i},
$$

where $D_{i}(t)$ is time passed in state $i$ in the interval time $[0, t]$.

$$
D_{i}(t)=\int_{0}^{t} \mathcal{I}_{\left\{L_{i-1} \leq X_{l}<L_{i}\right\}} d l .
$$

In the same manner, the long run expected productivity is:

$$
E P r_{\infty}=\lim _{t \rightarrow \infty}\left[\frac{E[\operatorname{Pr}(t)]}{t}\right]=\frac{E[\operatorname{Pr}(H)]}{E[H]} .
$$

The average of maintenance cost per unit of product can be defined as the ratio of the long run expected cost rate divided by the long run expected productivity rate.

$$
E C P_{\infty}=\frac{E C_{\infty}}{E P r_{\infty}}=\frac{E[C(H)]}{E[\operatorname{Pr}(H)]}
$$

This quantity can be estimated by using a simulation algorithm. However the estimation method could be computationally burdensome. An analytical calculation is investigated in the next paragraph.

\section{Analytical calculation}

It is shown in [3] and [6] that $\left(X_{t}\right)_{t \geq 0}$ describing the evolution of the system under the previous maintenance policy is:

- a regenerative process with regeneration times being the dates of replacement;

- a semi-regenerative process with semi-regeneration times being the inspection time $\left(T_{n}\right)_{n \subseteq \mathcal{N}}$.

The discret-time random process describing the system state at each inspection time $\left(Y_{n}=X_{T_{n}}\right)_{n \subseteq \mathcal{N}}$ is a Markov chain with continuous state space $[0, M)$. Its transition probability density function is:

$$
\begin{aligned}
P(d y \mid x) & =\bar{F}_{\beta, \alpha m(x)}(M-x) \delta_{0}(d y) \\
& +f_{\beta, \alpha m(x)}(y-x) \mathcal{I}_{\{y \geq y<M\}},
\end{aligned}
$$

where:

- $\delta_{0}$ is a Dirac mass function,

$$
\text { - } \bar{F}_{\beta, \alpha m(x)}(y)=\int_{y}^{+\infty} f_{\beta, \alpha m(x)}(u) d u
$$

It is also proved in [6] that this Markov chain $\left(Y_{n}\right)_{n \subseteq \mathcal{N}}$ is Harris ergodic with a unique invariant spread out probability distribution $\pi$ :

$$
\pi(d y)=a \delta_{0}(d y)+(1-a) b(y) d y,
$$


with

$$
\begin{aligned}
a & =\frac{1}{1+\int_{0}^{M} B(x) d x} \\
b(y) & =\frac{a}{1-a} B(y), \\
B(y) & =f_{\beta, \alpha m(0)}(y)+\int_{0}^{y} B(x) f_{\beta, \alpha m(x)}(y-x) d x .
\end{aligned}
$$

The equation (13) can be calculated by using Volterra technics, see [6].

Using these results, it is shown in [3] that the maintenance cost rate in equation (6) is written as the following:

$$
\begin{aligned}
E C_{\infty} & =\frac{E_{\pi}\left[C\left(T_{1}\right)\right]}{E_{\pi}\left(T_{1}\right)} \\
& =\frac{C_{i} E_{\pi}\left[N_{i}\left(T_{1}\right)\right]}{E_{\pi}\left(T_{1}\right)}+\frac{C_{p} E_{\pi}\left[N_{p}\left(T_{1}\right)\right]}{E_{\pi}\left(T_{1}\right)} \\
& +\frac{C_{c} E_{\pi}\left[N_{c}\left(T_{1}\right)\right]}{E_{\pi}\left(T_{1}\right)}+\frac{C_{d} E_{\pi}\left[d\left(T_{1}\right)\right]}{E_{\pi}\left(T_{1}\right)}
\end{aligned}
$$

where:

$$
\begin{aligned}
E_{\pi}\left[N_{i}\left(T_{1}\right)\right] & =1 \\
E_{\pi}\left[N_{p}\left(T_{1}\right)\right] & =P_{\pi}\left(M \leq X_{T_{1}^{-}}<L\right) \\
=\int_{[0, M]}\left[\bar{F}_{\beta, \alpha m(x)}(M-x)-\bar{F}_{\beta, \alpha m(x)}(L-x)\right] \pi(d x) & \\
E_{\pi}\left[N_{c}\left(T_{1}\right)\right] & =P_{\pi}\left(X_{T_{1}^{-}} \geq L\right) \\
& =\int_{[0, M[} \bar{F}_{\beta, \alpha m(x)}(L-x) \pi(d x) \\
E_{\pi}\left[d\left(T_{1}\right)\right] & =\int_{[0, M[} E_{\pi}\left[\int_{0}^{T_{1}} \mathcal{I}_{\left\{X_{s} \geq L\right\}} d s\right] \pi d(x) \\
& =\int_{[0, M[}\left[\int_{0}^{T_{1}} P_{\pi}\left(X_{s} \geq L\right) d s\right] \pi d(x) \\
& =\int_{[0, M[}\left[\int_{0}^{m(x)} \bar{F}_{\beta, \alpha s}(L-x) d s\right] \pi d(x) \\
E_{\pi}\left[\left(T_{1}\right)\right] & =\int_{[0, M[} m(x) \pi d(x)
\end{aligned}
$$

In the same manner, the average of maintenance cost per unit of product in equation (9) can be expressed as:

$$
\begin{aligned}
E C P_{\infty} & =\frac{E_{\pi}\left[C\left(T_{1}\right)\right]}{E_{\pi}\left[\operatorname{Pr}\left(T_{1}\right)\right]} \\
& =\frac{C_{i} E_{\pi}\left[N_{i}\left(T_{1}\right)\right]}{\sum_{i=1}^{N} S_{i} E_{\pi}\left[D_{i}\left(T_{1}\right)\right]}+\frac{C_{p} E_{\pi}\left[N_{p}\left(T_{1}\right)\right.}{\sum_{i=1}^{N} S_{i} E_{\pi}\left[D_{i}\left(T_{1}\right)\right]} \\
& +\frac{C_{c} E_{\pi}\left[N_{c}\left(T_{1}\right)\right]}{\sum_{i=1}^{N} S_{i} E_{\pi}\left[D_{i}\left(T_{1}\right)\right]}+\frac{C_{d} E_{\pi}\left[d\left(T_{1}\right)\right]}{\sum_{i=1}^{N} S_{i} E_{\pi}\left[D_{i}\left(T_{1}\right)\right]} .
\end{aligned}
$$

with

$$
\begin{aligned}
& E_{\pi}\left[D_{i}\left(T_{1}\right)\right]=\int_{[0, M[} E_{\pi}\left[\int_{0}^{T_{1}} \mathcal{I}_{\left\{L_{i}>X_{s} \geq L_{i-1}\right\}} d s\right] \pi d(x) \\
= & \int_{[0, M[}\left[\int_{0}^{m(x)}\left(\bar{F}_{\beta, \alpha s}\left(L_{i-1}-x\right)-\bar{F}_{\beta, \alpha s}\left(L_{i}-x\right)\right) d s\right] \pi d(x),
\end{aligned}
$$

for all $i=1,2, . . N$.

\section{NUMERICAL EXAMPLE}

The purpose of this section is to show how the proposed maintenance model can be used in maintenance optimisation of production deteriorating systems through a simple example.

Considering a single unit production system in which its degradation behavior is assumed to be described by a Gamma process with scale parameter $\alpha=1$ and shape parameter $\beta=5$. The nominal production capacity of the system is 100 products / unit of time $\left(S_{\text {nominal }}=100\right)$. The production capacity can be changed according to the degradation level of the system. More precisely:

- when the degradation level $X_{t}<20$, the system is in State 1 (normal operation state), production capacity is $S_{1}=S_{\text {nominal }}=100$;

- $20 \leq X_{t}<40$, the system is in state 2 , production capacity is $S_{2}=60$;

- $40 \leq X_{t}<60$ (state 3), production capacity is $S_{3}=40$;

- and when $60 \leq X_{t}$ (system fails), production capacity is $S_{4}=0$.

Assume that inspection, preventive and corrective maintenance cost are $C_{i}=2, C_{p}=90, C_{c}=100, C_{d}=100$.

\section{A. Inter-inspection time}

Firstly, inter-inspection time can be calculated by using equation (2). Fig. 3 represents the inter-inspection time function $m($.$) with respect to the degradation level of the$ system $x$ and the parameter $Q$. Obviously, an increase of the degradation level leads to a decrease of the inter-inspection time and the form of inter-inspection time function can be changed depending on the value of $Q$. Remember that only some particular forms are considered in [1], [6].

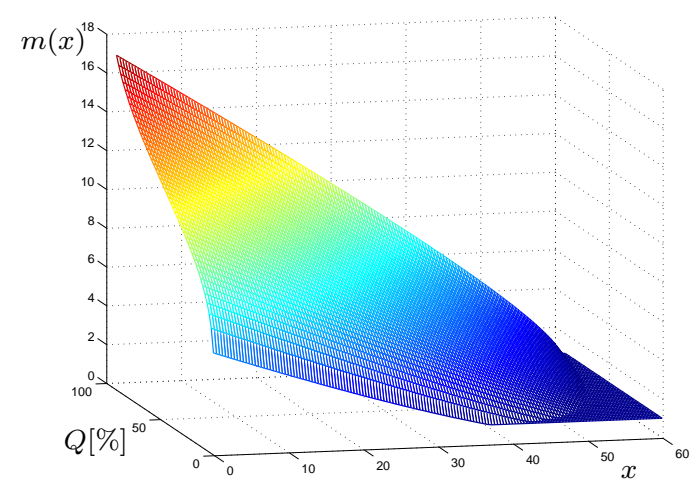

Fig. 3. Inspection scheduling function $\Delta T=m(x)$ as a function of $Q$ 
Fig. 4 and 5 show the inter-inspection time function for two particular cases $Q=1.5 \%$ and $Q=90 \%$ respectively.

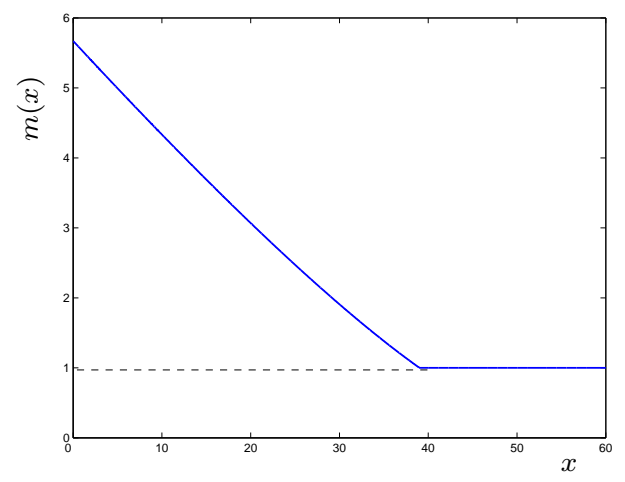

Fig. 4. $m(x)$ for $Q=1.5 \%$

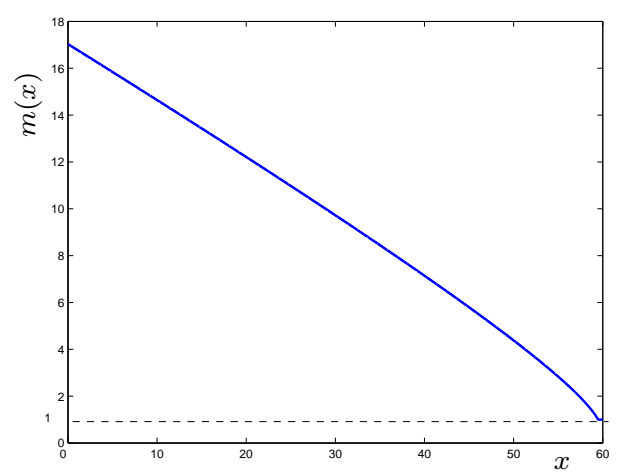

Fig. 5. $m(x)$ for $Q=90 \%$

\section{B. Cost based criterion policy}

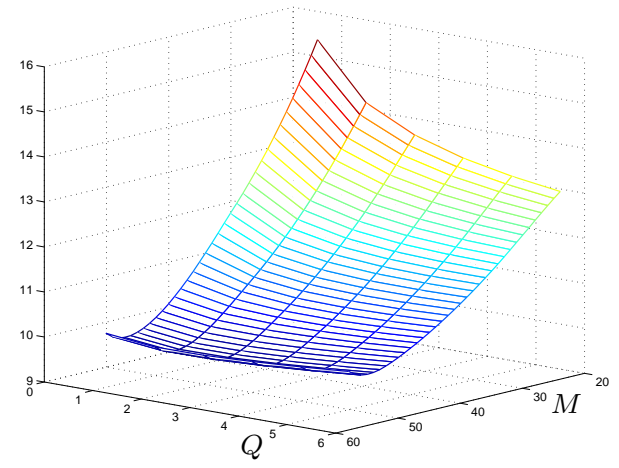

Fig. 6. Mean cost per unit of time as a function of $Q$ and $M$

The integration equations ((13), (16)-(19)) can be calculated by using numerical computing methods. Fig. 6 sketches the mean maintenance cost per unit of time for different values of the preventive maintenance threshold $M$ and the parameter $Q$.

A numerical optimisation scheme was used, the optimum values of the decision parameters are $M^{*}=51, Q^{*}=1.5 \%$ (see Fig. 7) for an optimal cost of $9.52\left(E C_{\infty}^{*}=9.52\right)$. The corresponding mean production capacity is $\operatorname{EPr}_{\infty}^{1}=63.81$.

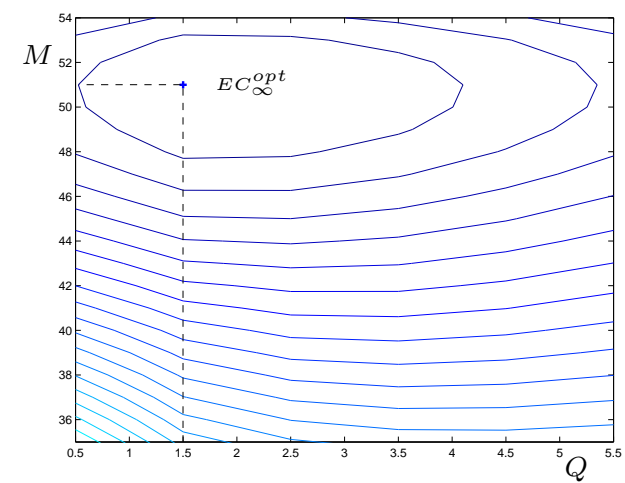

Fig. 7. Iso-level curves of mean cost per unit of time $E C_{\infty}$

Fig. 8 shows the relationship between the mean maintenance cost per unit of time and the mean production capacity. Each point corresponds to a couple of decision parameters $(M, Q)$. These results show that according to a demand on the production capacity level, relying on a production campaign for example, an optimal decision based on the minimal mean cost per unit of time can be reached.

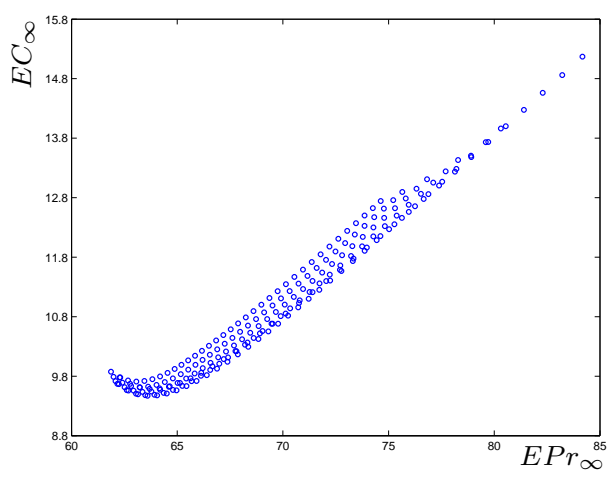

Fig. 8. Maintenance cost per unit of time as a function of production capacity. Each point corresponds to a couple of decision parameters $(M, Q)$

\section{Cost and productivity based criterion policy}

The evolution of the mean maintenance cost per unit of product and the iso-level curves are represented respectively in Fig. 9 and Fig. 10.

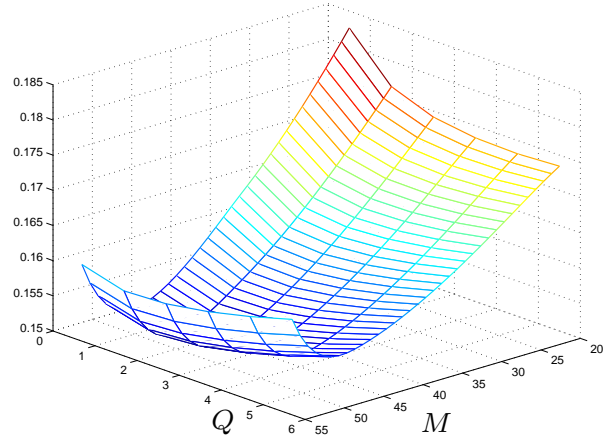

Fig. 9. Mean cost per product unit as a function of $Q$ and $M$

The optimal mean cost per unit of product is 0.1475 for 
$M^{*}=48, Q^{*}=1.5$ (see Fig. 10) and the corresponding mean production capacity and mean maintenance cost per unit of time are 65.17 and 9.61 respectively. We note that this cost rate is higher than that obtained from the cost based criterion policy however the production capacity in this case is higher. And it is not surprised that this mean cost per unit of product is lower than the ratio of $E C_{\infty}$ divided by $E P r_{\infty}^{1}$ obtained in the previous paragraph. More precisely, the cost saving is $1.14 \%$.

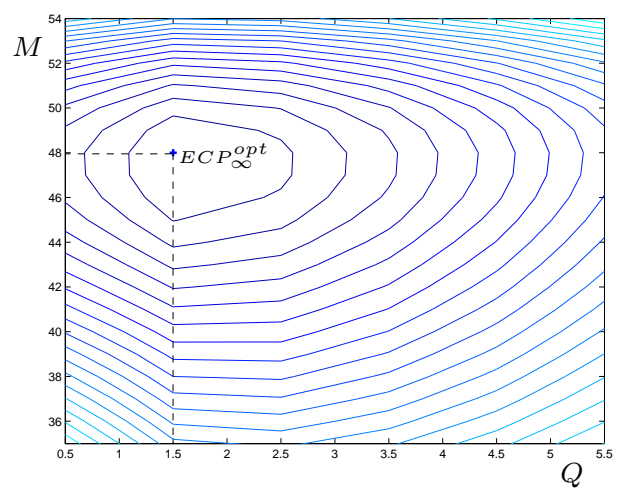

Fig. 10. Iso-level curves of mean cost per product unit $E C P_{\infty}$

The relationship between the mean maintenance cost per unit of product and the mean production capacity is sketched in Fig. 11. As we mentioned above, these results can be used to find an optimal decision for preventive maintenance activities, i.e. a couple of decision parameters $(M, Q)$, subject to a demand on the production capacity.

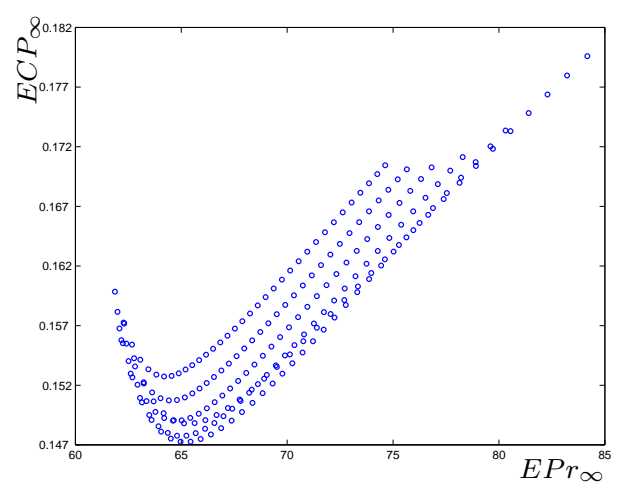

Fig. 11. Maintenance cost per unit of product as a function of production capacity. Each point corresponds to a couple of decision parameters $(M, Q)$

\section{CONCLUSIONS}

In this work, a maintenance model for condition based maintenance of deteriorating production systems is described. Both cost and productivity are taken into account in the optimisation procedure. This can help to find an adaptive optimal decision under a production level demand. When compared to the classical model in which only maintenance cost is considered, the proposed model provides better results. Quantile-based inspection (or RUL-based inspection) provides more insightful results on the aperiodic interinspection function that depends on the process parameters and doesn't have a fixed form ([1], [6]).

In order to compute the maintenance cost and production capacity, an analytical method based on semi-regeneration property and renewal theory is used. This leads to a simplification in the calculation procedure.

Our future research work will focus on the development this maintenance model to multi-unit system with different degradation behaviors.

\section{REFERENCES}

[1] BARKer, C., AND NEWBy, M. Optimal non-periodic inspection for a multivariate degradation model. Reliability Engineering and System Safety 94 (2009), 33-43.

[2] CUI, L., XIE, M., AND LOH, H.-T. Inspection schemes for general systems. IIE Transactions 39, 9 (2004), 817-825.

[3] Dieulle, L., Bérenguer, C., Grall, A., And Roussignol, M. Sequential condition-based maintenance scheduling for a eeteriorating system. European Journal of Operational Research 150 (2003), 451461.

[4] Do VAn, P., BArros, A., AND BÉrenguer, C. Importance measure on finite time horizon and application to markovian multi-state production systems. Journal of Risk and Reliability 222 (2008), 449461.

[5] Gebraeel, N., Lawley, M., Li, R., and Ryan, J. Residuallife distributions from components degradation signals : A bayesian approch. IIE Transactions 37 (2005), 543-557.

[6] Grall, A., Dieulle, L., BÉrenguer, C., And Roussignol, M. Continuous-time predective-maintenance scheduling for a deteriorating system. IEEE Transactions On Reliability 51 (2002), 141-150.

[7] Huynh, K., Barros, A., BÉrenguer, C., And CAstro, I. Value of condition monitoring information for maintenance decision-making. In Proc. Ann. Reliability \& Maintainability Symp (2010), pp. 543-548.

[8] Iung, B., Monnin, M., Voisin, A., Cocheteux, P., ANd Levrat, E. Degradation state model-based prognosis for proactively maintaining product performance. CIRP Annals - Manufacturing Technology 57 (2008), 49-52.

[9] KaWAUCHI, Y., AND RAUSAND, M. A new approach to production regularity assessment in the oil and chemical industries. Reliability Engineering and System Safety 75 (2002), 379-388.

[10] Khoury, E., Grall, A., And Brenguer, C. A comparison of rul-based and deterioration-based maintenance policies for gradually deteriorating systems. European Safety and Reliability Conference (ESREL) Congress, to appear (2010).

[11] Ponchet, A., Fouladirad, M., And Grall, A. Imperfect condition-based maintenance on a finite time span for a gradually deterioration system. European Safety and Reliability Conference (ESREL) Congress, Prague, Czech Republic 1 (2009), 559-566.

[12] SEYED, M. I., AND IZAK, D. Integrated maintenance and production control of a deteriorating production system. IIE Transactions 34.

[13] VAN NOORTWIJK, J. A survey of the application of Gamma processes in maintenance. Reliability Engineering and System Safety 94 (2009), $2-21$.

[14] YAng, Y., AND KLUtKe, G.-A. A distribution-free lower bound for availability of quantile-based inspection schemes. IEEE Transactions On Reliability 50 (2001), 419-421. 